\title{
The overlap operator as a continued fraction*
}

\author{
A. Boriçi ${ }^{\mathrm{a}}$, A. D. Kennedy ${ }^{\mathrm{a}}$, B. J. Pendleton ${ }^{\mathrm{a}}$, and U. Wenger ${ }^{\mathrm{b} \dagger}$

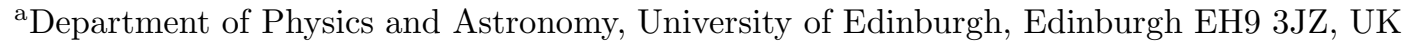 \\ ${ }^{\mathrm{b}}$ Theoretical Physics, Oxford University, 1 Keble Road, Oxford OX1 3NP, UK
}

We use a continued fraction expansion of the sign-function in order to obtain a five dimensional formulation of the overlap lattice Dirac operator. Within this formulation the inverse of the overlap operator can be calculated by a single Krylov space method where nested conjugate gradient procedures are avoided. We show that the five dimensional linear system can be made well conditioned using equivalence transformations on the continued fractions. This is of significant importance when dynamical overlap fermions are simulated.

\section{Introduction}

Let us start with the explicit form of the massive overlap Dirac operator with mass $\mu$,

$$
D(\mu)=\frac{1}{2}(1+\mu)+\frac{1}{2}(1-\mu) \gamma_{5} \operatorname{sgn}(H(-m)),
$$

where $H(-m)$ denotes some hermitian Dirac operator with mass $-m<0$ of the order of the inverse lattice spacing. One method to compute the action of the sign-function on a vector is by approximating the function by a rational polynomial [1],

$$
\operatorname{sgn}(x) \approx \operatorname{sgn}_{\{n, m\}}(x) \equiv \frac{P_{n}(x)}{Q_{m}(x)},
$$

where $P_{n}$ and $Q_{m}$ are irreducible polynomials of degree $n$ and $m$, respectively. The reason for using rational approximations is that for a given approximation error in the infinity-norm the required degree for rational approximations is usually much smaller than the one for polynomial ones, although both converge exponentially in their degree.

There are various methods to perform such an approximation. The methods differ in how they interpret the notion of the goodness of the approximation. One can use any definition of a

\footnotetext{
*Based on a talk by U. Wenger

${ }^{\dagger}$ Supported by British PPARC SPG
}

norm characterising the deviation of two functions from each other.

The infinity-norm, for example, is defined as the maximal error over a given interval $[a, b]$. There exists an algorithm (Remez algorithm [2]) to find the best (rational) approximation $r(x)$ to a function $f(x)$ with respect to the infinity-norm (see also the work of Zolotarjov [2, 3]). That is, the approximation $r(x)$ to a given function $f(x)$ is the rational function of a given degree that minimises the maximum value of the quantity $|f(x)-r(x)|$ over the interval $[a, b]$.

In order to approximate the sign-function by a rational polynomial we choose to find an approximation for a constant odd function over the interval $[\varepsilon, 1]$. To do so we approximate $\frac{1}{\sqrt{x}}$ on the interval $\left[\varepsilon^{2}, 1\right]$ to obtain the rational approximation $r(x)$ and consider the function $\operatorname{xr}\left(x^{2}\right)$.

The resulting approximation can then be written as

$$
\operatorname{sgn}_{\{2 n+1,2 m\}}(x)=x c_{0} \frac{\prod_{k=1}^{n}\left(x^{2}+p_{k}\right)}{\prod_{k=1}^{m}\left(x^{2}+q_{k}\right)}
$$

with real and positive $p_{k}$ and $q_{k}$. This factorised form is most stable for numerical evaluation.

One advantage of using rational polynomials is that one can use the partial fraction decomposition, e.g. for $n=m$,

$$
\operatorname{sgn}_{\{2 n+1,2 n\}}(x)=x\left(c_{0}+\sum_{k=1}^{n} \frac{c_{k}}{x^{2}+q_{k}}\right)
$$


which allows us to compute the action of the signfunction at roughly the cost of a single conjugate

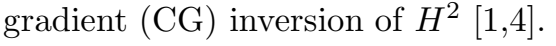

\section{Continued fractions}

It is well known that rational functions are closely related to finite (truncated) continued fractions which in turn play an important role in the analytic theory of rational functions [5],6]. Indeed, we can write any rational approximation to the sign-function as a continued fraction [7]

$$
\operatorname{sgn}_{\{2 n+1,2 n\}}(x)=\beta_{0} x+\frac{\alpha_{1}}{\beta_{1} x+\frac{\cdots}{\cdots+\frac{\alpha_{2 n}}{\beta_{2 n} x}}} .
$$

Then we observe that the inverse of eq. (5) is just the $(1,1)$-component of the Schur complement of a larger system

$$
\left(\begin{array}{ccccc}
\beta_{0} x & \sqrt{\alpha_{1}} & & & \\
\sqrt{\alpha_{1}} & -\beta_{1} x & & & \\
& & \ddots & & \\
& & & -\beta_{2 n-1} x & \sqrt{\alpha_{2 n}} \\
& & & \sqrt{\alpha_{2 n}} & \beta_{2 n} x
\end{array}\right) .
$$

Instead of inverting (4) using a two-level nested CG(-like) procedure we can now find the inverse of (6), i.e. its (1,1)-component, in one go, using just one CG(-like) search in a single higher dimensional Krylov space [7].

The operation naturally associated with rational functions is the multiplication and inversion which, of course, is most trivial. For the overlap operator, eq. (11), matters are not so simple since the additional term proportional to $\gamma_{5}$ does not commute with $\operatorname{sgn}(H)$ and its approximations. We can, however, still make use of some properties of (finite) continued fractions. The most important one is contained in the following theorem on equivalence transformations: Two continued fractions $\left\{\alpha_{0} ;\left\{\alpha_{1}, \beta_{1}\right\}, \ldots,\left\{\alpha_{n}, \beta_{n}\right\}\right\}$ and $\left\{\alpha_{0}^{\prime} ;\left\{\alpha_{1}^{\prime}, \beta_{1}^{\prime}\right\}, \ldots,\left\{\alpha_{n}^{\prime}, \beta_{n}^{\prime}\right\}\right\}$ are equivalent if and only if there exists a sequence of non-zero constants $c_{n}$ with $c_{0}=1$ such that

$\alpha_{n}^{\prime}=c_{n} c_{n-1} \alpha_{n}, \quad n=1,2,3, \ldots$,

$\beta_{n}^{\prime}=c_{n} \beta_{n}, \quad n=0,1,2, \ldots$.
This can most easily be seen by writing down the continued fraction after a transformation,

$$
\beta_{0}+\frac{c_{1} \alpha_{1}}{c_{1} \beta_{1}+\frac{c_{1} c_{2} \alpha_{2}}{c_{2} \beta_{2}+\frac{\cdots}{\cdots+\frac{c_{n-1} c_{n} \alpha_{n}}{c_{n} \beta_{n}}}}} .
$$

In fact the coefficients $c_{i}$ parametrise the equivalence class of a continued fraction corresponding to a given rational polynomial. It is important to note that although the equivalence transformation leaves the value of the continued fraction invariant it affects the spectrum and correspondingly the condition number of the larger system (6). One might therefore hope to find within an equivalence class of continued fractions the one with the smallest condition number.

\section{Application to the overlap operator}

It is easy to apply the equivalence transformation to an overlap operator in which the sign-function is approximated by a rational function and expanded as a continued fraction. In complete analogy to the previous section we express the linear system of the hermitian overlap Dirac operator $\gamma_{5} D(\mu) \psi=b$ as a simple five dimensional system $H^{(5)}(\mu) \Psi=\chi$ where $\Psi=\left(\psi, \phi_{1}, \ldots, \phi_{2 n+1}\right)^{T}, \quad \chi=(b, 0, \ldots, 0)^{T}$ are five dimensional fermion fields and $H^{(5)}(\mu)$ is a block tridiagonal matrix similar to (6) with $A_{+} \gamma_{5},-c_{1} k_{1} H,+c_{2} k_{2} H, \ldots,-c_{2 n+1} k_{2 n+1} H \quad$ on the diagonal, $\sqrt{c_{1} A_{-}}, \sqrt{c_{1} c_{2}}, \ldots, \sqrt{c_{2 n} c_{2 n+1}}$ with trivial Dirac and colour structure on the off diagonals and $A_{ \pm}=1 / 2(1 \pm \mu)$. The $k_{i}$ 's are uniquely determined by the rational approximation of the sign-function while the $c_{i}$ 's are free parameters describing the equivalence class of continued fractions associated with the given rational approximation.

We observe that an equivalence transformation on the continued fraction acts in a similar way to block Jacobi preconditioning. Indeed we can write $H^{(5)}(\mu)=C \cdot H^{(5)}\left(\mu ; c_{i}=1\right) \cdot C$ where $C$ is a block diagonal matrix having the four dimensional unit matrix $\mathbf{1}$ in the first block and 
$\sqrt{c_{i}} \cdot \mathbf{1}, i=1, \ldots, 2 n+1$ in the remaining ones. For the linear system under consideration this preconditioning comes for free since the first component of $\Psi$ and $\chi$ are not affected at all.

It turns out that within an equivalence class the condition number of $H^{(5)}, \kappa\left(H^{(5)}\right)$, can vary by orders of magnitude. An obvious choice for the equivalence transformation parameters is $c_{i}=$ $1 / k_{i}$, and indeed, with this choice the matrix is already well-conditioned. In order to find the optimal values for the $c_{i}$ in the sense of giving the lowest $\kappa\left(H^{(5)}\right)$ one can study the matrix in the free case where the spectrum can be calculated analytically. A non-linear minimisation of $\kappa\left(H^{(5)}\right)$ then yields a set of optimal values for the coefficients $c_{i}$.

How much is the effective gain in a practical application? In order to address this question we generated an ensemble of pure gauge field configurations with the Wilson gauge action at $\beta=5.9$ on $6^{4}$ lattices. We used the standard Wilson Dirac operator $H_{W}(-m)$ with $m=1.5$ as the input operator for the overlap and a $n=8$ rational approximation for the inverse square root on the interval $\left[\varepsilon^{2}, 1\right]=[0.00724,1]$. This generates a five dimensional operator with an extent of 18 in the fifth dimension. Then we calculated the inverse of the hermitian overlap operator $\gamma_{5} D(\mu)$ using the five and four dimensional formulation with different quark masses $\mu$ on a random vector.

Figure 11 shows the average number of $H_{W^{-}}$ matrix multiplications for the inversion in three cases: the pole approximation, eq. (4), ( $4 d$ overlap), the five dimensional operators $H^{(5)}$ with $c_{i}=1 / k_{i}(5 d$ matrix $)$ and $H^{(5)}$ with the optimal $c_{i}$ 's as obtained from the free field approximation ( $5 d$ matrix optimised). The results indicate that it is indeed possible to generate five dimensional systems which are well conditioned and that the obstacle of having large condition numbers for the five dimensional system can be avoided. For small quark masses the inversion seems to behave better than the one of the corresponding four dimensional system. This is of particular importance in the context of dynamical overlap fermion simulations where the inversion of the fermion matrix plays a crucial role [7 9]. Another advantage of

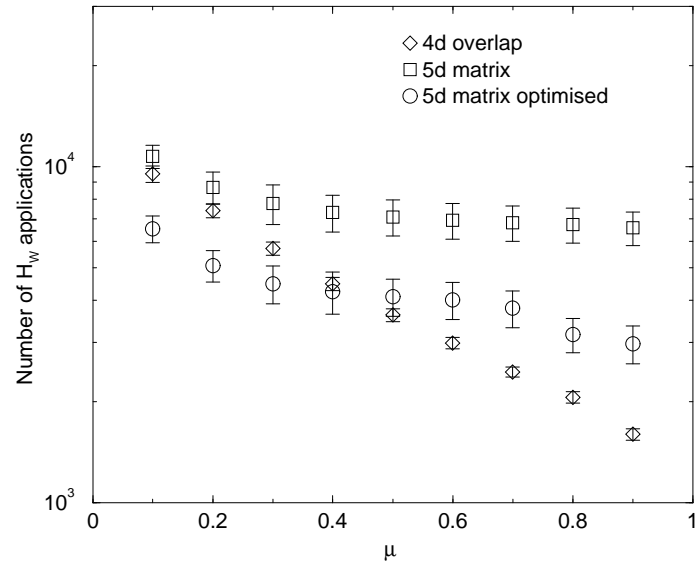

Figure 1. Number of $H_{W}$ applications for inverting the overlap operator in different four and five dimensional representations as a function of the quark mass $\mu$.

the five dimensional formulation using continued fractions is its obvious suitability for parallel computations.

\section{REFERENCES}

1. R. G. Edwards, U. M. Heller and R. Narayanan, Nucl. Phys. B540 (1999) 457, hep-lat/9807017.

2. P. Petrushev and V. Popov, Rational approximation of real functions (Cambridge University Press, 1987).

3. Th. Lippert et al., these proceedings.

4. H. Neuberger, Phys. Rev. Lett. 81 (1998) 4060, hep-lat/9806025.

5. H. Wall, Analytic theory of continued fractions (Chelsea Publishing company, 1948).

6. W. Jones and W. Thron, Continued fractions: analytic theory and applications, Vol. 11 (Addison-Wesley, 1980).

7. H. Neuberger, Phys. Rev. D60 (1999) 065006, hep-lat/9901003.

8. A. Bode et al., (1999), hep-lat/9912043.

9. R. Narayanan and H. Neuberger, Phys. Rev. D62 (2000) 074504, hep-lat/0005004. 